Rasim M. Alguliyev ${ }^{1}$, Nigar T. Ismayilova ${ }^{2}$

${ }^{1,2}$ Institute of Information Technology of ANAS, Baku, Azerbaijan

1asim@science.az, ${ }^{2}$ nigar@ iit.ab.az

\title{
PROSPECTS OF NEXT GENERATION DIGITAL LIBRARIES
}

\begin{abstract}
This paper analyzes different aspects of next generation digital libraries using contemporary information technologies - web technologies, multimedia opportunities, big data, data analyses, linguistic and technologies. It considers advantages of intellectual digital libraries, their realization possibilities and prospects.
\end{abstract}

Keywords: intellectual digital libraries, data mining, mobile digital libraries, bibliometrics, common e-library environment, multimedia technologies.

\section{Introduction}

In the modern world of information abundance and content diversity, the main tasks facing the e-libraries in Azerbaijan are to bring the treasure of knowledge into the virtual environment and to serve as a source of high-level information in the information war benefiting from the modern web technologies, multimedia and Wikipedia technologies. At the same time, through the e-library, it is possible to digitalize the information, films, records, and audio records, including the manuscripts in different alphabets (Cyrillic, Latin, Arabic, etc.) reflecting the history and culture of Azerbaijan and stored in museums and collections, as well as, to form a national information portal, and to provide the use of this resource by the international community and the Azerbaijanis living in various countries.

As a result of the rapid development of modern information and communication technologies and deep penetration of computer science into the library study and information sciences, the process of the formation of the new generation of e-libraries is dynamically developing. This article analyzes the features and advantages of next generation of e-libraries, and casts the light on scientific and technological challenges of existing e-libraries.

\section{E-libraries and web-technologies}

As the capabilities of Web-technologies are expanding and collective intelligence is developing, the process of improving the quality of service delivered to e-library users will continue through the development of an interactive dialogue with them (Figure 1). At the same time, as a result of certain problems of the users, gathering around the information resources, discussing and giving feedback, the e-libraries are turning into a social network environment. Accordingly, various social groups are formed in e-libraries for the users' interests, age, and activity and so on.

The introduction of Web technologies (Web 1.0, Web 2.0, Web 3.0, etc.) in e-libraries creates many opportunities for both librarians and users [3]:

- Improved interface is offered for users. E-library users are the people with different psychological conditions, knowledge and skills. Therefore, each user must be provided with appropriate, effective and interactive interface. Improvement of the user interfaces through Web technology significantly increases the comfort of their use;

- Blogs and Wiki-technologies is a system providing the users to share ideas and knowledge. Wiki-technologies and blogs are very supportive in organizing the work in e-libraries, such as, implementation of collective work, creating courses or training corps with the participation of all interested parties (lecturers, students, etc.) and dynamic teaching platforms;

- RSS (Really Simple Syndication) ensures users to receive information about current news without visiting the specific sites; 
- Multimedia files. Web-technology is the best platform for the distribution and accessibility of multimedia files offering variety of ways to demonstrate multimedia materials;

- Social networks. E-library-information system and social networking technologies provide more reliable and higher quality working principle of joint activity of not only information resources, but also the human resources. Using the social network infrastructure, theelibraries are forming the vital elements as e-library fund, user categories, profiles and so on with the user intervention;

- The users of e-libraries can easily send various resources (photos, video, audio, etc.) to other users and provide comprehensive search engine through the bookmarks and tagging.

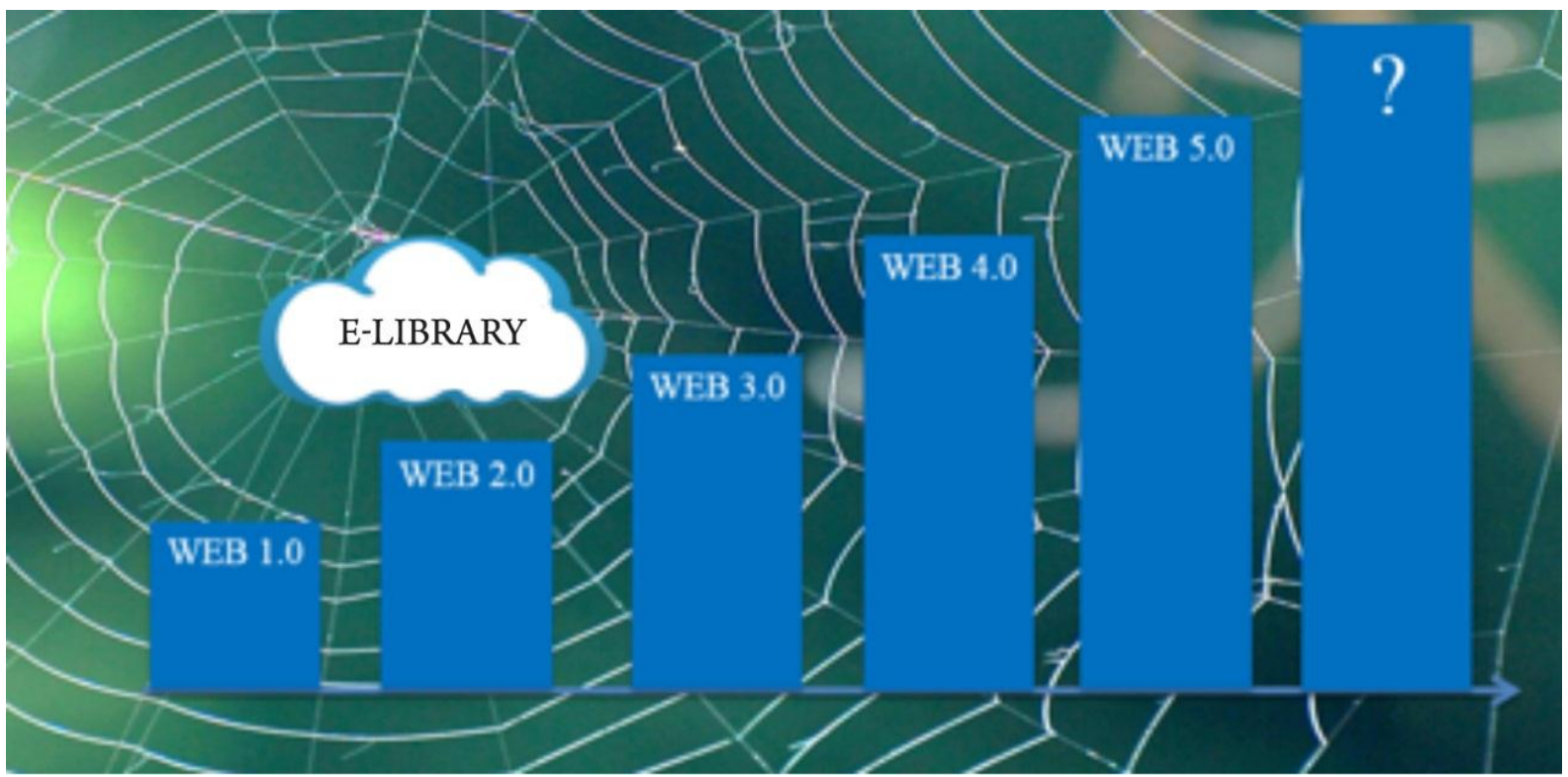

Figure 1. Web-technologies and e-libraries

\section{The integration of e-libraries}

Although there are currently a large number of various e-libraries at various organizations educational institutions, research centers and government agencies, the coordination between them or unit standards and so on do not exist. Therefore, the unification of e-libraries in a single technological platform and the deployment of their databases on a single server is very important. The integration of e-library environment as an integral part of e-government with other systems e-science, national terminology system, national search engine, national biometric identification system, and their coordination will create great opportunities for users (Figure 2). For instance, when a user is able to automatically view the explanation of the term he/she came across while reading any book in the national terminology system, it prevents the time loss and provides reliable and accurate information to a user. The integration with the biometric identification system perfectly resolves the user authorization problem. Furthermore, although anti-plagiarism systems are used at some enterprises, high level national digital content is not available for it yet. If the databases of e-libraries are collected in a single server, subsequently, the content problem of the anti-plagiarism system will be solved. 


\section{The application of big data and cloud technologies in e-Libraries}

Currently, cloud technologies are widely used for data storage and processing in a virtual environment. At the same time, as a result of the introduction of cloud technologies in e-libraries and the creation of user-clouds, the information about the use of resources is gathered. This analysis and processing of this information management provide ample opportunities for the implementation of the intelligent management of e-libraries [4].

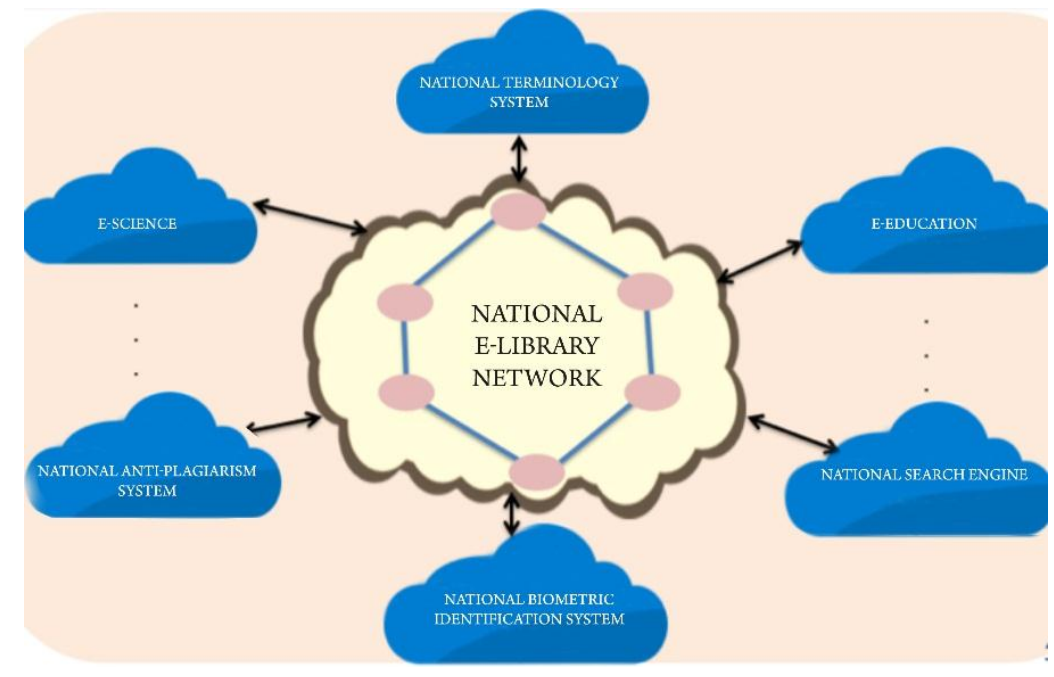

Figure 2. National e-libraries Network

As a result of the analysis of relations between the library users and the resources based on various indicators is the most useful resources of e-library, and the most active users can be identified. In this case, obtained information contributes to define from the useless resources and the passive users of the library, which is very important for the organization of the library.

Defining the scope of interests and behavior of the passive users based on the same principle, certain steps can be taken to increase their level of activity. In addition, the resources can be clustered by the destinations or types of the users, based on their workplace, profession and so on, and their interaction can be identified [5].

Data mining technologies, such as clustering, classification, grouping and visualization are used in e-libraries to obtain the necessary unknown knowledge out of large volume of data [6]. Data mining has a great impact on the organization of information in e-library, optimization of automated data processing, improvement of the quality of information services and intelligent management process (Figure 3 ).

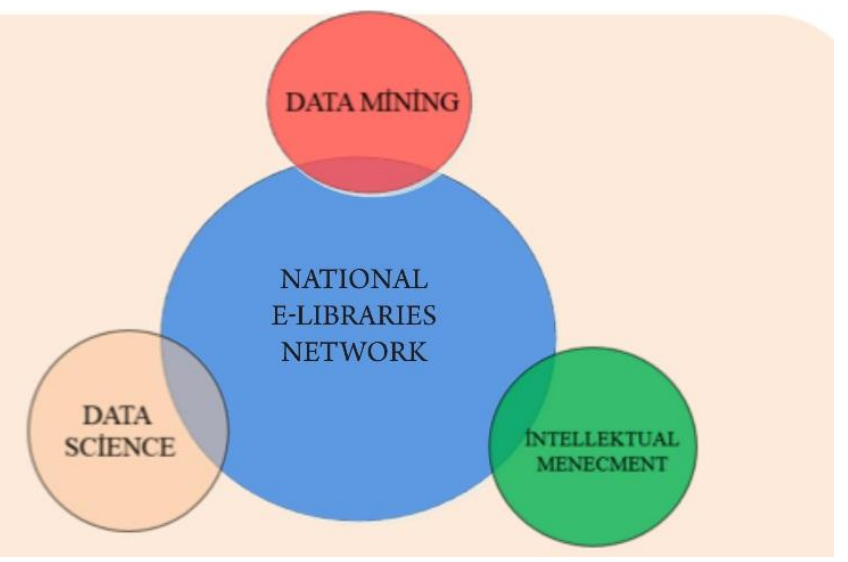

Figure 3. E-library intellectualization technologies 
Meanwhile, data mining technologies are applied for the classification of similar users according to their profile and keywords and for the clustering of related services. Hence, the users in the same cluster are more likely to be provide $\mathrm{d}$ with the appropriate services [7-8].

As an object of large-scale data studies, large e-libraries containing millions of digital books can be used to ensure the extraction of hidden knowledge and the intelligent management of relations of the users with the resources [9]. Since the modern e-libraries offer a large number of online resources and services, the librarians must use technologies for processing and improvement of digital cultural heritage, metadata, visualization technologies, 3D models of movable and immovable cultural heritage artifacts, new methods of search and storage, in other words, they should use analytical software through the application of data science. In addition to finding answers to the questions "What happened?" and "Why it happened?" with the application of data science, more accurate, understandable and meaningful knowledge can be detected. Moreover, as a result of extracting and analyzing the needed information out of the data available in e-library network using data analysis capabilities, new information resources in certain direction, form and context can be shaped. New generation e-libraries benefitting from the capabilities of Data Science will be capable to create new resources along with the available ones, provided to the readers.

\section{Providing information security of e-libraries}

Providing information security of e-libraries should be implemented at different levels (Figure 4): plagiarism and anti-plagiarism technology as a source of information, data privacy and content filtering and sanitization to ensure the use of different age groups, information security mechanisms to protect the database of e-libraries should be applied.

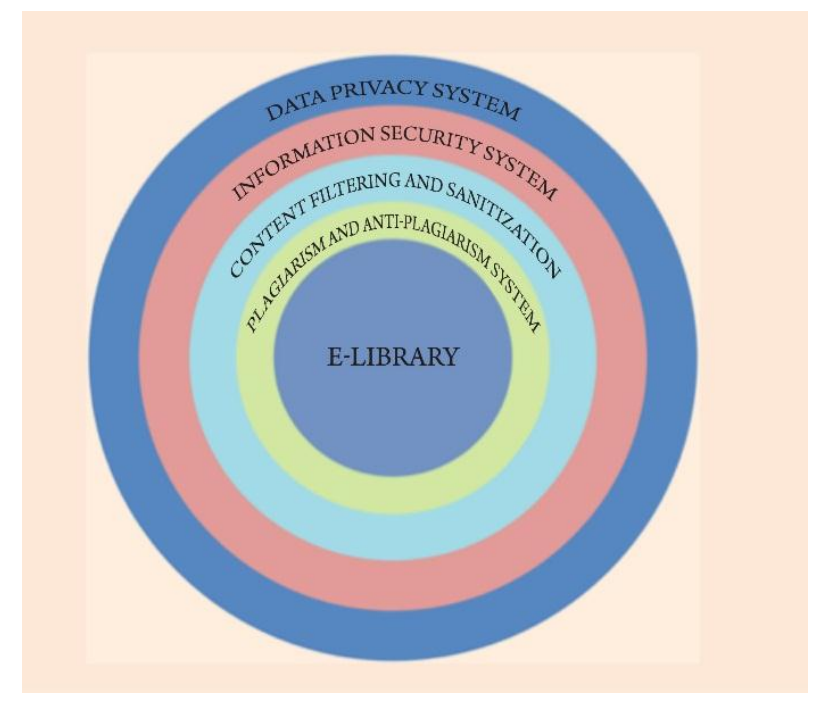

Figure 4. Levels of information security in e-libraries

Bank account of a person, realized operations, health information and the information about the library use is private data. To this end, data sanitization techniques should be used. E-libraries, data sanitization techniques are applied to avoid the disclosure of confidential information to a wider audience, namely storing the information sufficient for the use of third parties and deleting other data (concealment). Taking into account the personal data protection in e-library environment, including the content availability in online environment, the sanitization according to some features, including different age groups $(0+, 6+, 12+, 18+$ etc. $)$ is today's requirements [10].

Poor information security of libraries can cause the disclosure of personal data, the loss of database information and so forth due to attacks or other failures. They, in turn, can lead to distrust of authors and content providers, and even can cause difficulties for e-library owners and economic loss [11]. 
Different security requirements are available in e-libraries. Content provider must ensure protection of intellectual property, provision of conditions for the use of content. A user, in turn, should also be confident about the security of the information stored in e-library.

Since the e-libraries are often open to users, they are exposed to attacks of hackers and cybercriminals. In this regard, the use of biometric technology would be more reasonable. The use of biometric technologies replaces the e-registration by the physical registration; furthermore, the introduction of biometric technologies (fingerprints, palm, hand, voice, face, signature recognition, retina scanning etc.) in e-libraries serves to more perfect data protection [12].

\section{Intelligent e-libraries}

Emergence of e-libraries creates great opportunities for the education of disabled people and their normal integration into the society and positioning in the public. Currently, different technologies - screen reader programs, audio-books, e-books and physical books and the technology converting the information resources into Braille alphabet have been developed around the world to ensure visually impaired people to use e-libraries [13]. Text-to-speech technologies are also available to ensure the use of e-libraries by visually impaired people. At the same time, text-to-speech technologies, understanding the writing and sign language, and the methods for converting text into sign language and vice versa should be developed to facilitate dialogue between the users and the e-library. Moreover, the application of "smart" monitors in e-libraries is very significant for both deaf and blind users. With the help of "smart" monitors will enable understanding the language of gestures and signs; and remote control by swiping, selecting the needed information and the implementation of other gestures, and controlling the behavior in front of the screen will save the resources.

Understanding and visualization of the text is one of the key issues for the absolute use of e-library by the disabled users. Autism patients, people with mental disorders may take advantage of text illustration, animation, 3D, 4D, 5D, 6D and other technologies to ensure the use of e-libraries. Such technologies may evoke an interest to the user; the user understands the text and perceives the needed information in this way.

\section{Mobile e-libraries}

Many libraries develop mobile versions of their web sites to offer more opportunities for their users. They offer information in all formats for small screen about library services and collections, search opportunities in the library catalogues, portable demonstration, subject guides, e-journals. Different e-library systems offer mobile web sites for users, for example, OPLINMobile, AirPac and others. [14-15]. OverDrive digital media service collaborates with 7500 libraries to offer audiobook collections, e-books, video and music files; in addition, MobiPocket and Adobe Reader programs can be downloaded in portable devices. The users can be given news, announcements, important information via short messages sent by e-libraries, or their inquiries can be replied. At the same time, various mobile technologies have been developed for the library staff to remotely implement their obligations outside of the library [16]. With the development of mobile web technologies, the e-libraries are capable to organize video-conference between a librarian and users; moreover, different services as using the e-library contents through e-books and audio books without web-browsers are also available.

Modern libraries use RFID (Radio-Frequency Identification) technologies to ensure the safety of self-service and resources. In addition, RFID tag enables the library to send the information about the material to the mobile device via the wireless connection. Smart monitors can offer the user other materials according to the books he/she read, and send him/her notifications about the events of the library and brief information about the books (feedback, pictures, etc.). Not only RFID tag, but also other "smart" tools contribute to the improvement of the quality of library services with the introduction of the Internet of Things. 


\section{E-libraries and linguistic technologies}

In the globalizing world, as a result of the development of the Internet, the e-libraries not only exceed the limits, but also eliminate the language barrier to some extent.

Gathering collections from different countries, regions and cultures enable discovery of information and ideas in a broader context, information availability on a global scale, protection of cultural heritage, and the establishment of more perfect economy [17].

To establish multilingual e-libraries professionals of various fields (computer science, library science, natural language processing, art, etc.) must work together. A wide application of linguistic technologies in e-libraries will lead to the establishment of cooperation between and among the nations and to the integration of different cultures and mentalities as a result of the automated conversion of the content from one language to another.

Meanwhile, providing the automated conversion of various alphabets (Cyrillic, Latin, Arabic, Greek, etc.) into each other with the introduction of transliteration system will grant more frequent use of e-libraries. This problem is especially important for Azerbaijan. Hence, as a result of changing the alphabet several times for various reasons, citizens experience great difficulty in reading the library resources written in different alphabets.

\section{The convergence of e-libraries}

As the modern e-library is the information system itself, it lays the ground for the implementation of metrics of the sciences such as informetrics, bibliometrics, scientometrics and altmetrics, sociometrics, and psychometrics since it becomes a social network of the users (Figure 5). Evaluation of e-libraries, its statistics, studying its dynamics, classification of e-libraries by different criteria, identifying the activity indicators of the users according to their age, profession and location through the bibliometric technologies are the key factors of forming the knowledge society.

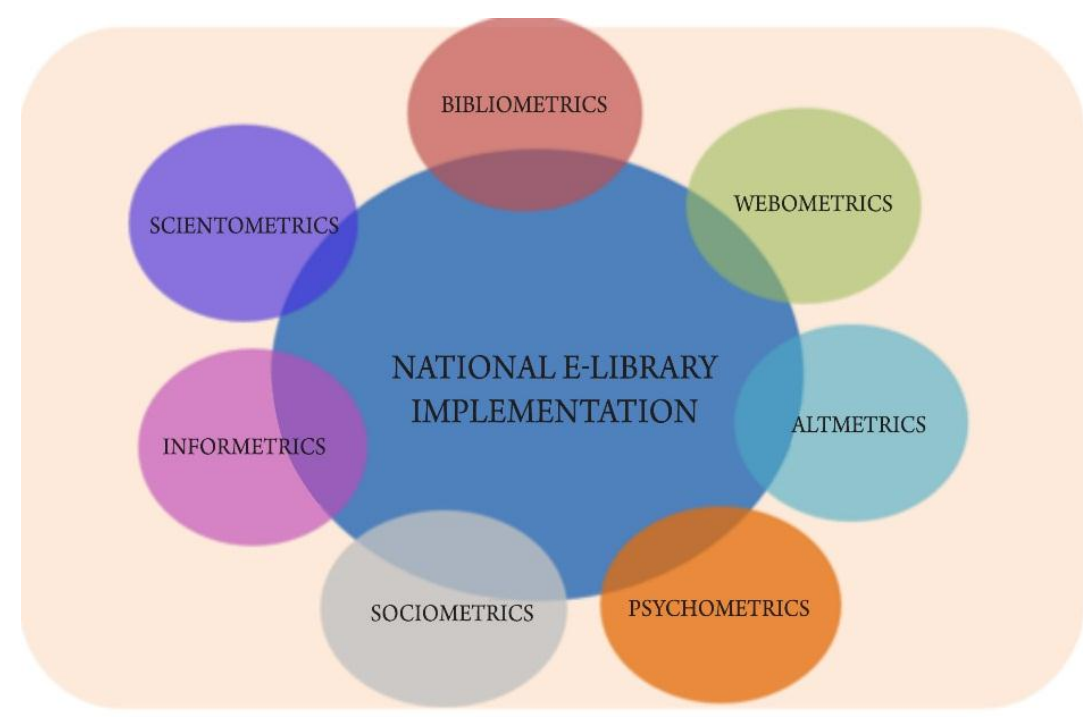

Figure 5. E-library Analysis Technology

\section{Conclusion}

As a result of introducing modern technology in the next generation e-libraries, completely eliminating location and time problems, ensuring security of resources and personal data of users, and detecting social networks in e-libraries the results of the studies aimed at the improvement of the library management, offering intelligent library services will enhance the quality of e-library services for users including physically impaired people. With the introduction of modern information technologies, formation of the intellectual e-libraries will contribute to satisfy the information needs of the society, at the highest level, by paying low costs and spending less time. 


\section{References}

1. Chowdhury G., Chowdhury S. Introduction to Digital Libraries, Facet Publishing, 2003.

2. Greenstein D. Next-generation digital libraries, http://vala.org.au/vala2002/2002pdf/-01Grnstn.pdf

3. SastryH.G., Reddy L.C. Significance of Web 2.0 in Digital Libraries // International Journal on Computer Science and Engineering, 2010, vol.2, no 6, pp. 2208-2211.

4. Alguliyev R., Alakbarov R., Hashimov M., Mammadov E. The perspectives of cloud technology implementationin digital library //2015,Sylwan, vol. 159, no 3, pp.97-108.

5. Alguliyev R., Mammadov E., Jafarov J., Ismayilova N., Mammadova R. "Extraction of Social Networks in Modern Digital Library Environment // Economics \& Sociology, vol. 2015, 8, no 1, pp. 308-317.

6. Kim H. Developing Semantic Digital Libraries using Data Mining Techniques, Dissertation presented to the graduate School of the University of Florida in Partial Fulfillment of the Requirements for the degree of Doctor of Philosophy, 2005, p.126.

7. Bin Ch. Study on Data Mining in Digital Libraries. Information Computing and Applications. Communications in Computer and Information Science, vol.392, 2013,pp. 282-291.

8. Kovacevic V. Devedzic V. Pocajt M. Using Data Mining to Improve Digital Library Services // The Electronic Library, 2010,vol. 28, issue 6, pp.829-843.

9. Gordon-Murnane L. Big Data: A Big Opportunity for Librarians // Online, 2012, vol.36, no 5.

10. WathamS.D., Vimal V.R. Design and Implementation of Data Sanitization Technique For Effective Filtering With Enhanced Medical Support System in Cloud Architecture Diagram // International Journal of Emerging Technology and Advanced Engineering, 2013,vol.3, issue 12, pp. 471-473.

11. Tyrwainen W. Concepts and a Design for Fair Use and Privacy DRM // D-lib Magazine, 2005, vol.11, no 2.

12. Kumbargoudar R. Biometric Security Technology for Libraries // Indian Streams Research Journal, 2013, 2 (12), 6 p.

13. Golub K. Digital libraries and the blind and visually impaired, 4th CARNet Users Conference - CUC, Zagreb (Croatia), 25-27 September 2002, p. 10.

14. http://oplin.ohio.gov/oplin-mobile-app-saga

15. http://www.bradford.ac.uk/library/my-library/mobile-catalogue/

16. Kroski E. Move with the Mobile Web: Libraries and Mobile Technologies. http://eprints.rclis.org/12463/1/mobile_web_ltr.pdf

17. Yang Ch. Cross-lingual Thesaurus for Multilingual Knowledge Management // Decision Support Systems, 2010, vol. 45, pp. 596-605. 